\title{
Garcinol Blocks the Reconsolidation of Multiple Cocaine-Paired Cues after a Single Cocaine-Reactivation Session
}

\author{
Amber B Dunbar ${ }^{1,2}$ and Jane R Taylor ${ }^{*, 1,2,3}$ \\ 'Division of Molecular Psychiatry, Department of Psychiatry, Yale University, New Haven, CT, USA; '2Department of Psychology, Yale University, \\ New Haven, CT, USA; ${ }^{3}$ Interdepartmental Neuroscience Program, Yale University, New Haven, CT, USA
}

\begin{abstract}
Manipulations of memory reconsolidation can interfere with the ability of a drug-paired cue to drive drug-seeking behavior. However, the typical reconsolidation paradigm that reactivates the memory through the presentation of the cue (conditioned stimulus (CS)) only interferes with the memory of the reactivated CS while leaving other drug-paired CSs intact and able to continue driving drug-seeking behavior. Here, we used a novel unconditioned-stimulus (US) reactivation paradigm to interfere with the ability of multiple cues to drive drug-seeking behavior after just one reactivation and treatment session. Rats were trained to self-administer cocaine, during which time each active lever press resulted in an i.v. cocaine infusion paired with one of two cues that alternated within each session. The drug memory was later reactivated with either i.v. or i.p. cocaine presentation in the absence of any cue. The histone acetyltransferase (HAT) inhibitor garcinol or vehicle was injected following US reactivation to impair reconsolidation. Rats were later tested on cue-induced reinstatement to both cues. Garcinol administered after either i.v. or i.p. cocaine reactivation significantly decreased cue-induced reinstatement to both cues, indicative of reconsolidation impairment. In addition, garcinol administered in the absence of reconsolidation or at a $6 \mathrm{~h}$ delay when the memory should be restabilized had no effect on reinstatement, further suggesting that garcinol's effects on reinstatement are through reconsolidation-based mechanisms. Our results demonstrate that a US-reactivation paradigm may be preferable to traditional CS-reactivation paradigms for treating disorders that involve multiple CS-US associations and support investigations of garcinol as a therapeutic pharmacological agent.

Neuropsychopharmacology (2017) 42, I884-1892; doi:I0.1038/npp.2017.27; published online 12 April 2017
\end{abstract}

\section{INTRODUCTION}

The theory of memory reconsolidation posits that upon recall, stored memories can destabilize into a labile state, during which time they can be modified before being stored again in long-term memory. Manipulations of memory reconsolidation show promise as a potential treatment for psychopathologies including addiction (Taylor et al, 2009; Torregrossa and Taylor, 2013), a disorder in which one unconditioned stimulus (US; ie, the drug) is associated with a number of conditioned stimuli (CSs; eg, paraphernalia, the environment, internal states, and so on). However, the typical reconsolidation paradigm, in which an amnestic agent is administered following CS memory retrieval, inhibits the memory of only the CS that was reactivated, whereas the memory of another CS paired with the same US remains unaffected (Doyère et al, 2007; Monsey et al, 2016b). Impairment of only one CS-US association may be ineffective for treating psychiatric disorders such as drug addiction that are characterized by multiple CS-US associations.

* Correspondence: Dr JR Taylor, Department of Psychiatry, Yale University School of Medicine, 300 George Street, New Haven, CT 065 II, USA, Tel: 203974 7727, Fax: 2037857357.

E-mail: jane.taylor@yale.edu

Received 2 July 2016; revised 28 January 2017; accepted 30 January 2017; accepted article preview online 7 February 2017
Therefore, developing a paradigm that can impair the memory of multiple CSs paired with a single US may better inform the translational potential of reconsolidation-based therapies. In a fear conditioning paradigm, reactivating an aversive memory through presentation of the US rather than the CS, followed by intraamygdalar protein synthesis inhibition, can effectively disrupt the association of multiple CSs paired with a single US (Debiec et al, 2010; Díaz-Mataix et al, 2011). In addition, in a model of cocaine selfadministration, repeated CS-extinction sessions immediately following US retrieval impaired reinstatement to the extinguished CS and to a nonextinguished CS (Luo et al, 2015). The ability of a single injection of an amnestic agent following US retrieval on interfering with the reconsolidation of multiple drug-paired cues has yet to be tested and holds great promise for potential clinical implementation.

The present study provides a direct test of the efficacy of administering an amnestic agent directly after a US memory retrieval session to attenuate reinstatement of drug-seeking behavior in a drug self-administration model. Rats were trained to lever press for i.v. cocaine infusions that were paired with two distinct CSs that alternated during the session. The US memory was subsequently reactivated through either i.v. or i.p. injections of cocaine, followed by systemic delivery of the nonspecific histone acetyltransferase (HAT) inhibitor garcinol (Balasubramanyam et al, 2004). 
Garcinol can be administered systemically in both humans and rats and has been shown to impair reconsolidation in fear conditioning and cocaine self-administration when administered after CS reactivation (Maddox et al, 2013a; Monsey et al, 2016b). Finally, cue-induced reinstatement was assessed for both cues.

We report that a single reactivation and treatment session can impair the reconsolidation of multiple drug-paired cues, as garcinol significantly attenuated reinstatement to both CSs in a reactivation-dependent manner indicative of reconsolidation inhibition. Importantly, the effect of garcinol on reconsolidation occurred whether the US was reactivated through the same or a different route of administration as during training, which may enhance the translational efficacy of the US-reactivation paradigm.

\section{MATERIALS AND METHODS}

Detailed materials and methods can be found in the Supplementary Information.

\section{Subjects}

Male Sprague Dawley rats (250-275g) were individually housed on a $12 \mathrm{~h}$ light cycle. All procedures were conducted in accordance with the policies of the Yale University Institutional Animal Care and Use Committee and conformed to National Institutes of Health Guidelines on the Care and Use of Laboratory Animals.

\section{Surgery}

Rats were implanted with a chronic, indwelling catheter in the right jugular vein. The catheter tubing connected to a cannula mounted on the back of the animals. Animals were allowed to recover for 5-7 days.

\section{Behavioral Apparatus}

Behavioral procedures took place in sound-attenuating operant chambers. Context A contained a syringe pump that was connected to one end of a spring tether to deliver i.v. cocaine infusions to rats. Context B contained altered visual, olfactory, and tactile cues. All procedures except for memory reactivation occurred in context A. Memory reactivation (or control procedures) occurred in context B (i.v. reactivation) or the home cage (i.p. reactivation).

\section{Behavioral Procedures}

Animals underwent acquisition of cocaine selfadministration for 10-12 days in $1 \mathrm{~h}$ sessions. Each active lever press resulted in one infusion of cocaine $(0.5 \mathrm{mg} / \mathrm{kg})$ paired with two conditioned CSs that alternated within each self-administration session. Inactive lever presses were recorded but had no outcome. Rats next underwent 8-10 days of $1 \mathrm{~h}$ lever extinction sessions. No CS presentations or cocaine infusions were available during the extinction sessions.

US memory retrieval sessions (or control manipulations) occurred $72 \mathrm{~h}$ after the final extinction session. In groups that underwent i.v. memory reactivation, rats were placed in context $\mathrm{B}$ for $6 \mathrm{~min}$ in total. After a $2 \mathrm{~min}$ acclimatization period, 2 i.v. cocaine infusions $(0.5 \mathrm{mg} / \mathrm{kg})$ were administered with a 2 min intertrial interval, and rats remained in the box for an additional $2 \mathrm{~min}$. The CSs were never presented during the reactivation session. In the i.v. control group without memory reactivation, rats underwent identical procedures but received saline infusions instead of cocaine. Rats were immediately removed from the boxes and returned to their home cages. In rats that underwent i.p. memory reactivation, animals received injections of cocaine $(10 \mathrm{mg} /$ $\mathrm{kg}$, i.p.) in their home cages. In the i.p. control group, rats received an equivalent volume of saline (i.p.) in their home cages.

Following reactivation or control treatment, rats were injected with garcinol $(10 \mathrm{mg} / \mathrm{kg}$ in $100 \%$ DMSO, $0.10-0.14 \mathrm{ml}$, i.p.) or vehicle ( $100 \% \mathrm{DMSO}, 0.10-0.14 \mathrm{ml}$, i. p.). This dose has been demonstrated to interfere with reconsolidation in other paradigms (Maddox et al, 2013a; Monsey et al, 2016b). Garcinol was injected $60 \mathrm{~min}$ after reactivation, $30 \mathrm{~min}$ later than other studies using garcinol (Maddox et al, 2013a; Monsey et al, 2016b) to account for the half-life of cocaine (Nayak et al, 1976). One subset of rats, the delayed-treatment control group, received garcinol injections $6 \mathrm{~h}$ after memory reactivation in order to assess whether the effects of garcinol are specific to reconsolidation. Injections given $6 \mathrm{~h}$ after retrieval should be well past the window of lability for manipulating memories during destabilization period, and amnestic agents should have no effect on reconsolidation at this time point (Sorg, 2012; Tronson and Taylor, 2007).

At $72 \mathrm{~h}$ after reactivation, animals were tested on cueinduced reinstatement. The reinstatement session consisted of four 15 min blocks. In Block A each active lever press resulted in the contingent presentation of CS1, and in Block $B$ each active lever press resulted in the contingent presentation of CS2. Blocks A and B were presented two times each in a counterbalanced order. No cocaine infusions were administered during reinstatement.

\section{Statistical Analysis}

Data for self-administration, extinction, and reinstatement were analyzed with repeated measure analyses of variance (rm-ANOVAs) across days. Following significant rm-ANOVAs, planned comparisons (one-way ANOVAs) were performed. To examine the effects on the two cues separately, numbers of active lever presses on reinstatement day for CS1 and CS2 were analyzed in $2 \times 2$ ANOVAs.

\section{RESULTS}

\section{Effect of Garcinol on Reconsolidation after US Reactivation with I.V. Cocaine}

To examine whether systemic garcinol can impair reconsolidation after US memory retrieval, rats received i.v. cocaine infusions to reactivate the US memory followed by garcinol injections, and cue-induced reinstatement was later assessed (Figure 1a). Across cocaine self-administration, no differences were found between rats that would be injected with vehicle $(N=12)$ or garcinol $(N=13)$ on number of cocaine infusions (Supplementary Figure S1a), active lever presses, or 
a

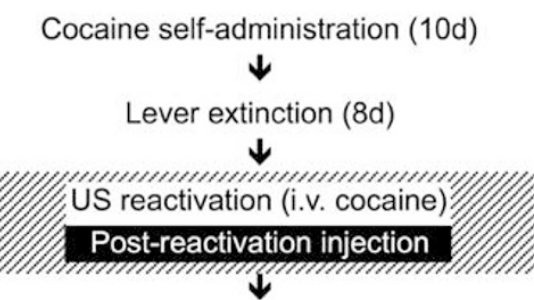

Cue-induced reinstatement test
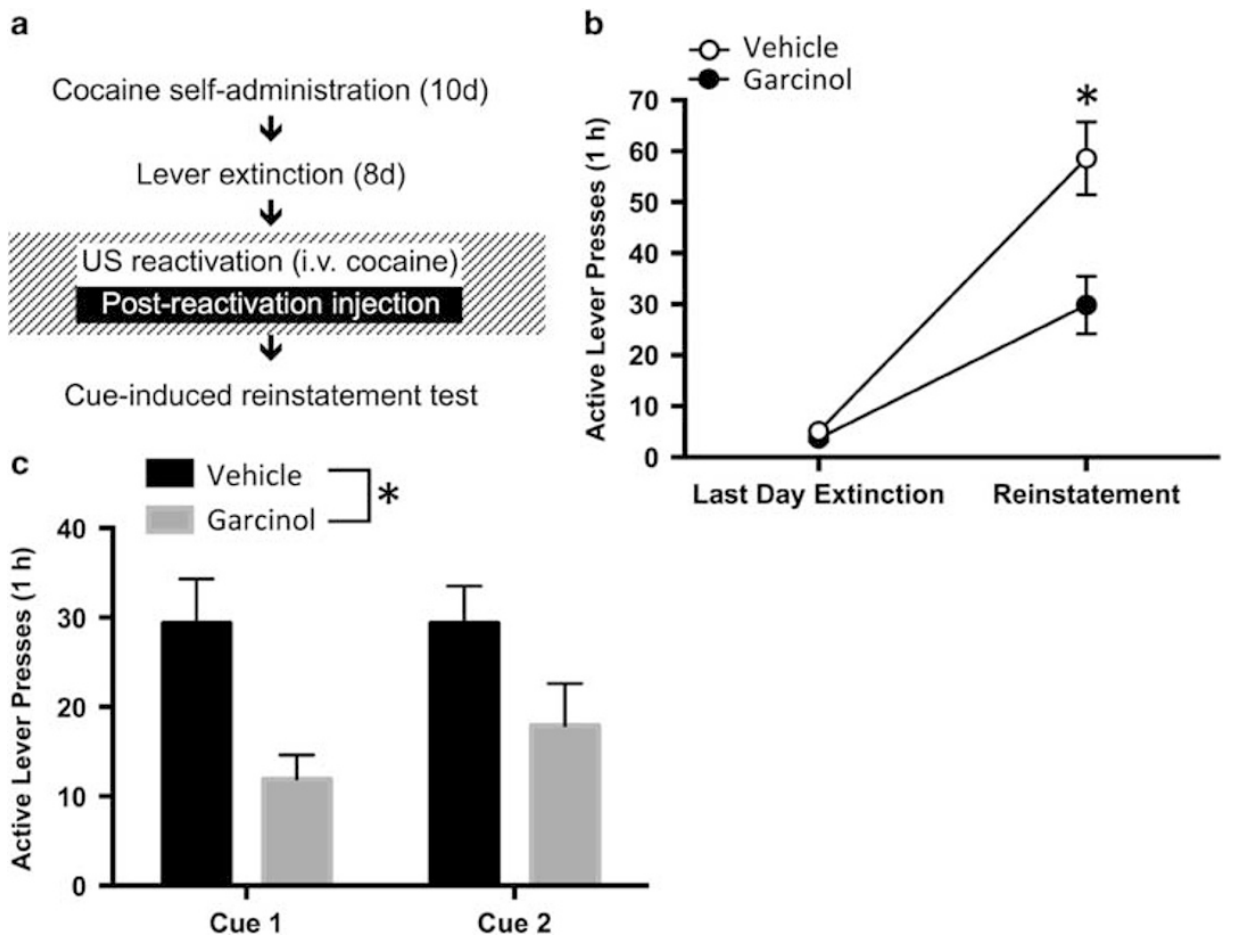

Figure I Systemic garcinol administered after US reactivation with i.v. cocaine impairs reconsolidation. (a) Schematic representation of the experimental procedures. White background: context A; checkered background: context B. (b) Active lever presses on the last day of extinction and on the cue-induced reinstatement test. (c) Active lever presses on the cue-induced reinstatement test for each cue. Vehicle $=\mid 2$, garcinol $=\mid 3$; *Statistically significant $(p<0.05)$.

inactive lever presses $(p s>0.05)$. Similarly, there were no between-group differences across lever press extinction on number of active lever presses (Supplementary Figure S1b) or inactive lever presses ( $p s>0.05$ ).

A main effect of session (last day of extinction $v s$ reinstatement $)$ was found $\left(F_{(1,23)}=73.59, p<0.001\right)$, such that rats pressed the active lever significantly more on reinstatement $(\mathrm{M}=44.26 \pm 4.50)$ as compared with the last day of extinction $(M=4.43 \pm 0.55)$. A main effect of drug (vehicle $v s$ garcinol) was also found $\left(\mathrm{F}_{(1,23)}=11.70\right.$, $p=0.002$ ), but the main effect of drug was qualified by a significant session by drug interaction (Figure 1b; $\left.\mathrm{F}_{(1,23)}=8.67, p=0.007\right)$. There were no differences in active lever presses between groups on the last day of extinction $(p>0.05)$, whereas on reinstatement day animals that had received garcinol $(M=29.85 \pm 6.24)$ pressed the active lever significantly less than animals that had received vehicle $\left(\mathrm{M}=58.67 \pm 6.50 ; \mathrm{F}_{(1,23)}=10.24, p=0.004\right)$. There were no significant main effects or drug by session interaction on inactive lever presses ( $p s>0.05)$.

When active lever presses on reinstatement day were separated by cue, a main effect of drug was found (Figure 1c; $\left.\mathrm{F}_{(1,23)}=10.24, p=0.004\right)$, such that rats pressed the active lever less regardless of cue when they had received garcinol $(M=14.92 \pm 3.12)$ as compared with vehicle $(M=29.33 \pm 3.25)$. No effects were found for inactive lever presses $(p s>0.05)$. Overall, these data indicate that garcinol administered following i.v. cocaine memory retrieval inhibits reconsolidation to multiple drug-paired cues, consistent with the hypothesis.

\section{Effect of Garcinol on Cue-Induced Reinstatement When Administered after I.V. Saline}

To assess whether the effects of garcinol on cue-induced reinstatement require memory reactivation, control rats received i.v. saline infusions before garcinol or vehicle injection and were later tested on reinstatement (Figure 2a). Across self-administration, no differences were found between rats that would be injected with vehicle $(N=5)$ or garcinol $(N=5)$ on total number of cocaine infusions (Supplementary Figure S2a), active lever presses, or inactive lever presses $(p s>0.05)$. Comparably, no betweengroup differences were found across extinction on number of active lever presses (Supplementary Figure S2b) or inactive lever presses $(p s>0.05)$. A significant main effect of session (last day of extinction $v s$ reinstatement) was found on number of active lever presses $\left(\mathrm{F}_{(1,8)}=24.67, p=0.001\right)$, such that all rats pressed the active lever significantly more on reinstatement $(\mathrm{M}=38.2 \pm 7.47)$ as compared with extinction $(\mathrm{M}=4.1 \pm 0.65)$. No main effect of drug or drug by session interaction on active lever presses was obtained (Figure 2b; $p s>0.05)$. Similarly, no main effects or drug by session interaction on inactive lever presses was obtained ( $p s>0.05$ ). In addition, no significant effects were found when active lever presses were separated by cue (Figure 2c; $p s>0.05$ ). These data indicate that garcinol has no effect on cueinduced reinstatement in the absence of memory retrieval and confirms the above finding that post-US-reactivation garcinol has reactivation-dependent effects on memory reconsolidation. 
a

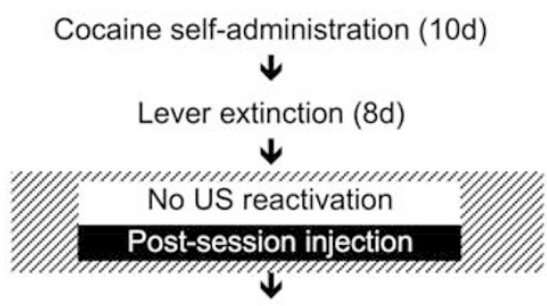

Cue-induced reinstatement test

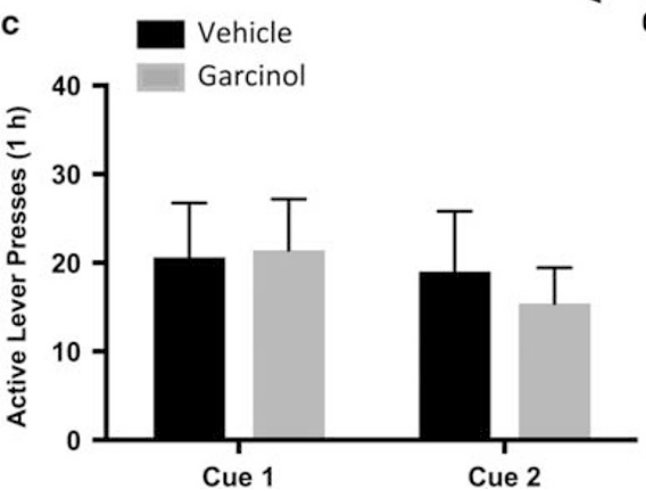

b

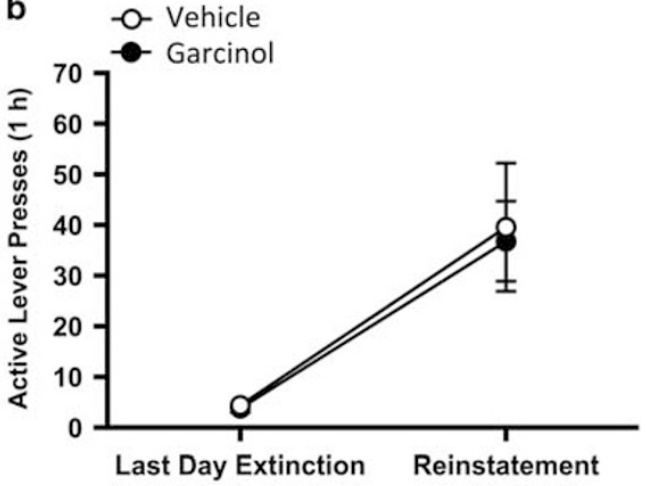

Figure 2 Garcinol administered after i.v. saline does not affect cue-induced reinstatement. (a) Schematic representation of the experimental procedures. White background: context A; checkered background: context B. (b) Active lever presses on the last day of extinction and on the cue-induced reinstatement test. (c) Active lever presses on the cue-induced reinstatement test for each cue. Vehicle $=5$, garcinol $=5$.

\section{Effect of a Delayed Injection of Garcinol on Cue-Induced Reinstatement}

An additional control group was run to verify that garcinol would have no effect when administered after reconsolidation should have already occurred. Rats underwent US memory reactivation with i.v. cocaine, were injected with garcinol or vehicle $6 \mathrm{~h}$ after memory retrieval, and were later tested on cue-induced reinstatement (Figure 3a). No differences emerged across self-administration for rats that would later be treated with vehicle $(N=6)$ or garcinol $(N=6)$ on total number of cocaine infusions (Supplementary Figure S3a), active lever presses, or inactive lever presses ( $p s>0.05)$. Similarly, no between-group differences were found across extinction on number of active lever presses (Supplementary Figure S3b) or inactive lever presses ( $p s>0.05)$. A main effect of session (last day of extinction $v s$ reinstatement) was obtained $\left(\mathrm{F}_{(1,10)}=15.82, p=0.03\right)$, such that rats pressed the active lever significantly more on reinstatement $(\mathrm{M}=35.50 \pm 7.24)$ as compared with extinction $(M=7.00 \pm 1.78)$. No main effect of drug or session by drug interaction on active lever presses was obtained (Figure 3b; $p s>0.05$ ). Similarly, no main effects or session by drug interaction on inactive lever presses were obtained ( $p s>0.05$ ). In addition, when active lever presses were split by cue, no significant effects were found (Figure 3c; ps $>0.05)$. These data indicate that garcinol administered outside of the postretrieval time period of memory lability has no effect on reinstatement, confirming that garcinol has temporally specific effects consistent with the reconsolidation time course.

\section{Effect of Garcinol on Reconsolidation after US Reactivation with I.P. Cocaine}

An additional experiment was conducted in order to examine whether garcinol's ability to impair reconsolidation after US memory retrieval requires that the cocaine be administered through the same route during both training and reactivation. Instead of i.v. infusions during reactivation, rats received i.p. cocaine infusions to reactivate the US memory followed by garcinol injections and were later tested on cue-induced reinstatement (Figure 4a). Across selfadministration training, no differences were found between rats that would later receive post-reactivation injections of vehicle $(N=8)$ or garcinol $(N=9)$ on total number of cocaine infusions (Supplementary Figure S4a), active lever presses, or inactive lever presses $(p s>0.05)$. Similarly, no between-group differences were obtained across extinction on number of active lever presses (Supplementary Figure $S 4 b)$ or inactive lever presses $(p s>0.05)$.

A main effect of session (last day of extinction $v s$ reinstatement) on number of active lever presses was found $\left(\mathrm{F}_{(1,15)}=125.40, p<0.001\right)$, such that rats pressed the active lever significantly more on reinstatement $(M=66.11 \pm 5.64)$ as compared with extinction $(\mathrm{M}=7.63 \pm 1.08)$. A main effect of drug (vehicle $v s$ garcinol) was also found on active lever presses $\left(F_{(1,15)}=8.97, p=0.009\right)$, but this main effect was qualified by a significant session by drug interaction (Figure $4 \mathrm{~b} ; \mathrm{F}_{(1,15)}=8.41, p=0.011$ ). On the last day of extinction, both groups exhibited comparable active lever pressing behavior $(p>0.05)$, but on reinstatement day, the rats that received post-reactivation garcinol $(M=49.22 \pm 7.74)$ pressed the active lever significantly less than saline-treated rats $\left(\mathrm{M}=83.00 \pm 8.21 ; \mathrm{F}_{(1,15)}=8.96\right.$, 
a

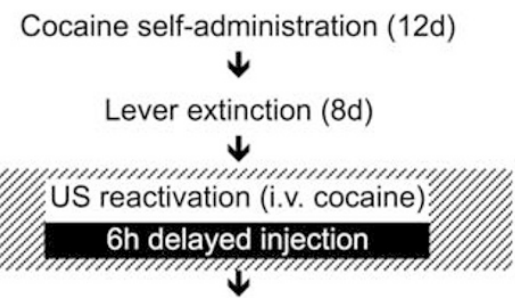

Cue-induced reinstatement test

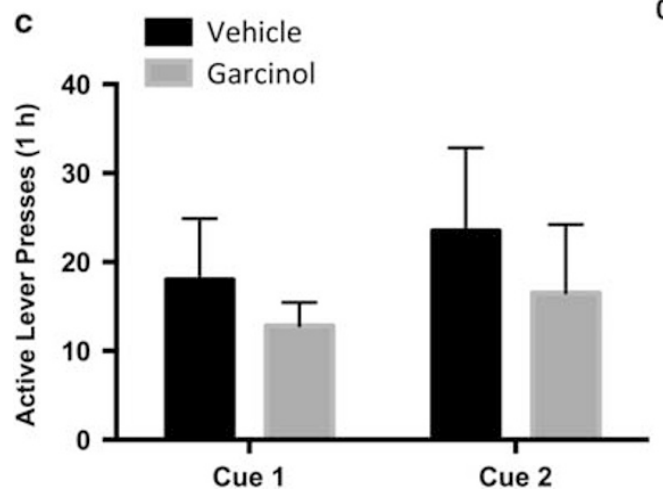

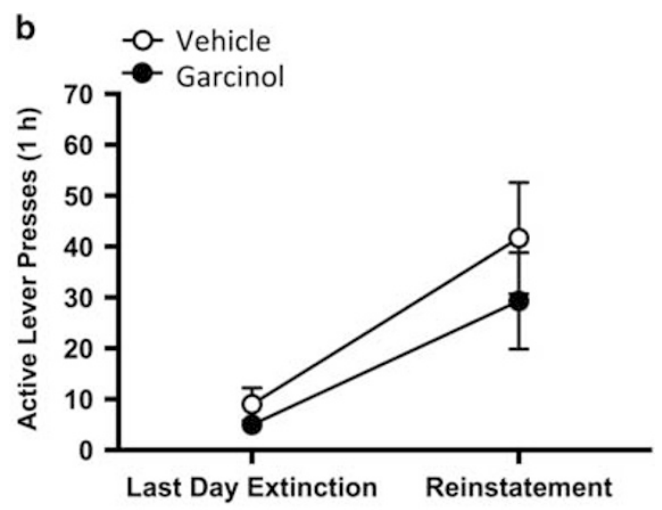

Figure 3 Garcinol does not affect cue-induced reinstatement when administered $6 \mathrm{~h}$ after US reactivation with i.v. cocaine. (a) Schematic representation of the experimental procedures. White background: context A; checkered background: context B. (b) Active lever presses on the last day of extinction and on the cue-induced reinstatement test. (c) Active lever presses on the cue-induced reinstatement test for each cue. Vehicle $=6$, garcinol $=6$.

a

Cocaine self-administration (10d)

$$
\downarrow
$$

Lever extinction (8d)

$\downarrow$

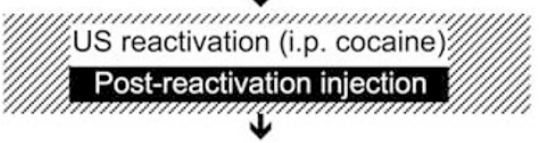

Cue-induced reinstatement test

C

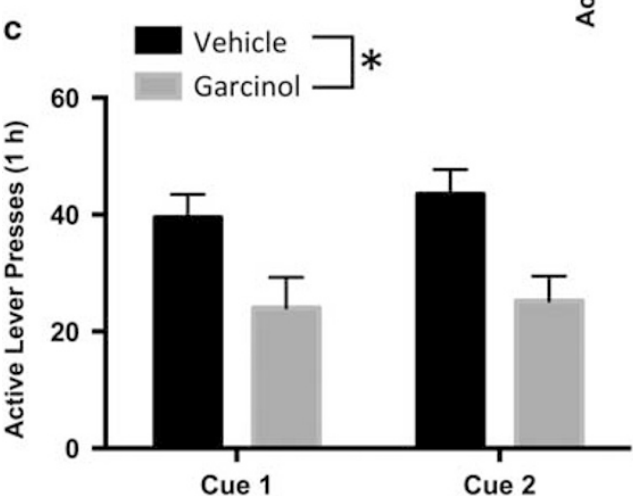

b - $\quad$ - Vehicle

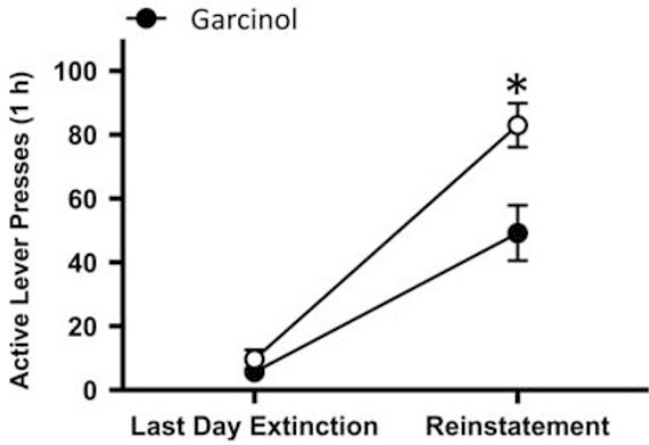

Figure 4 Systemic garcinol administered after US reactivation with i.p. cocaine impairs reconsolidation. (a) Schematic representation of the experimental procedures. White background: context A; checkered background: context B. (b) Active lever presses on the last day of extinction and on the cue-induced reinstatement test. (c) Active lever presses on the cue-induced reinstatement test for each cue. Garcinol $N=8$, vehicle $N=9$; *Statistically significant $(p<0.05)$.

$p=0.009)$. No main effects or session by drug interaction on inactive lever presses was obtained ( $p s>0.05)$.

When the number of active lever presses on reinstatement was separated by cue, a main effect of drug was obtained
(Figure 4c; $\mathrm{F}_{(1,15)}=8.96, p=0.009$ ), such that garcinoltreated rats $(M=24.61 \pm 3.87)$ pressed the active lever significantly less than vehicle-treated rats $(M=41.5 \pm 5.11)$. These data indicate that garcinol administered after i.p. 
a

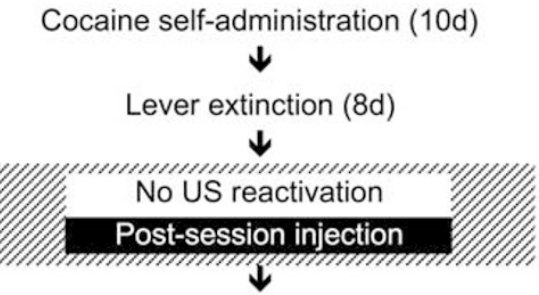

Cue-induced reinstatement test b

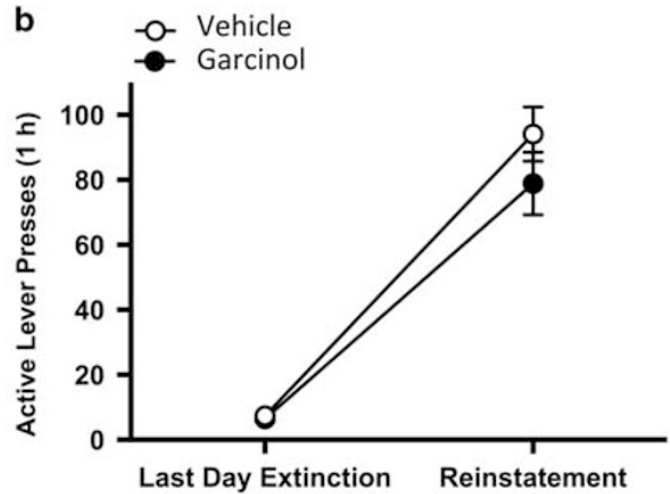

Last Day Extinction Reinstatement

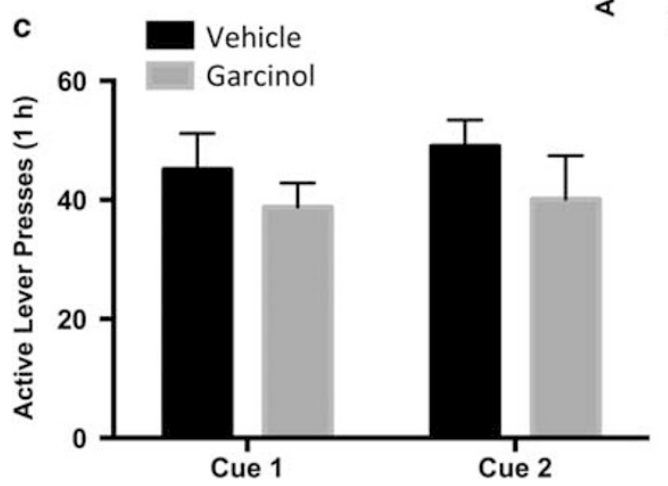

Figure 5 Garcinol administered after i.p. saline does not affect cue-induced reinstatement. (a) Schematic representation of the experimental procedures. White background: context A; checkered background: context B. (b) Active lever presses on the last day of extinction and on the cue-induced reinstatement test. (c) Active lever presses on the cue-induced reinstatement test for each cue. Vehicle $=9$, garcinol $=9$.

cocaine memory reactivation inhibits reconsolidation to multiple drug-paired cues, mirroring the effects of garcinol administered after i.v. cocaine memory reactivation.

\section{Effect of Garcinol on Cue-Induced Reinstatement When Administered after I.P. Saline}

A control group was run to verify that garcinol has no effect on reinstatement when administered in the absence of reactivation in the i.p. paradigm. In lieu of memory reactivation, rats received i.p. saline injections in the home cage followed by garcinol or vehicle injections. Cue-induced reinstatement was later assessed (Figure 5a). Across selfadministration, no differences were found between rats that would later receive vehicle $(N=9)$ or garcinol $(N=9)$ on total number of cocaine infusions (Supplementary Figure S5a), active lever presses, or inactive lever presses ( $p s>0.05)$. Similarly, no differences were found between groups across extinction on number of active lever presses (Supplementary Figure S5b) or inactive lever presses $(p s>0.05)$. A significant main effect of session (last day of extinction $v s$ reinstatement) was obtained $\left(\mathrm{F}_{(1,16)}=176.28, p<0.001\right)$, such that rats pressed the active lever significantly more on reinstatement $(\mathrm{M}=86.50 \pm 6.36)$ as compared with extinction $(\mathrm{M}=11.83 \pm 2.50)$. No additional main effects or session by drug interaction were obtained for active (Figure $5 b$ ) or inactive lever presses ( $p s>0.05$ ). Similarly, when the active lever presses on reinstatement were separated by cue, no significant effects were found (Figure $5 c$; $p s>0.05$ ). These data indicate that garcinol administered following i.p. saline injection has no effect on cue-induced reinstatement. As there was no effect found in the delayed-injection control group after i.v. reactivation, this indicates that garcinol does not exhibit nonspecific effects on reinstatement. Therefore, no delayed-injection group was carried out after i.p. cocaine reactivation.

\section{DISCUSSION}

\section{Summary of Findings}

The present results provide the first demonstration that a novel US-reactivation paradigm can interfere with the ability of multiple drug-paired cues to drive drug-seeking behavior after a single reactivation and treatment session, in contrast to most other paradigms that utilize only one drug-paired CS. Specifically, administration of the naturally occurring HAT inhibitor garcinol after US reactivation attenuated cueinduced reinstatement to multiple cocaine-paired CSs. Importantly, the effect of garcinol was dependent both upon memory reactivation and upon administration during the postretrieval window of memory lability. In addition, US reactivation was successfully achieved through either i.p. or i.v. administration of cocaine, suggesting that US administration during treatment does not require the same route of administration as during drug use. This has valuable translational implications, as cocaine addicts ingest the drug through several different routes of administration.

Importantly, the pharmacological approach demonstrated in the present studies requires only a single garcinol treatment session. The only other study that has interfered with the reconsolidation of multiple drug-paired cues utilized 12 treatment sessions (Luo et al, 2015). In addition, those US retrieval-extinction sessions still necessitated the presentation of at least one drug-paired CS during 195 min extinction sessions over each of the 12 days (Luo et al, 2015). Our current findings demonstrate that the strength of multiple drug-paired CSs can be weakened in the absence of any CS 
presentation using a much shorter US procedure. The present approach may, thus, be preferable to other reconsolidation-based addiction treatment models and should be further investigated as a method for rapidly impairing the ability of drug-associated cues to induce relapse to drug-seeking behavior.

\section{Possible Mechanisms of Reconsolidation Impairment in the US-Reactivation Paradigm}

Our novel US-reactivation paradigm in which garcinol impairs the reconsolidation of multiple drug-paired CSs improves upon the standard CS-reactivation paradigm that inhibits only the directly reactivated CS (Monsey et al, 2016b). The effect of the US-reactivation paradigm on multiple cues may result from a number of reasons. One possibility is that the conventional paradigm including the reactivation of one CS may recruit the neural correlates of only that specific CS-US association, thereby rendering only that CS-US association amenable to memory impairment through administration of a post-reactivation amnestic agent. Conversely, reactivation of the US may recruit the neural circuits related to the association between the US and all linked CSs, thus allowing for memory impairment of all CS-US associations following administration of a postreactivation amnestic agent. Future work may further explore this possibility. The latter paradigm tested here may be more useful in the clinical setting, as it would more effectively allow for disruption of the entire memory network associated with the psychopathological memory.

Although we believe the most parsimonious explanation may be that garcinol's amnestic effects were due to alterations in multiple CS-drug memories, other explanations are possible. We do not believe that inhibition of a common context-cocaine memory was altered by garcinol because rats underwent multiple lever press extinction sessions in the drug self-administration context in which no cocaine or CSs were presented. Rats that did not display full extinction were excluded from analysis (see Supplementary Information). Because extinction is highly context specific (see Bouton et al, 2006), it is unlikely that US presentations of i.v. or i.p. cocaine conducted in a separate context would involve recall of the extinguished original context and subsequently result in inhibiting a single common context-drug memory without affecting the CS memories. Regardless, if US reactivation paradigms could reduce contextual components (or other memory processes such as configural representations) while simultaneously reducing craving associated with multiple drug cues, such a paradigm would be advantageous clinically. Future studies could empirically address whether explicit cocaine-context representations could be altered by US-reactivation and reconsolidation mechanisms and also whether distinct CSs associated with cocaine in distinct contexts could be targeted by amnestic agents under US retrieval conditions in separate contexts to precisely define the nature of the representations themselves, though we are not aware of such parallel studies in fear conditioning paradigms. The key is that associative relationships are bidirectional and that accessing the relationship via the US may be more effective in treating humans, because we have much less direct knowledge of what acts as a cue in each individual (eg, interoceptive cues, exteroceptive cues, other people, and so on). It is unlikely that all cues can be accessed in laboratory situations; however, we can both access and administer cocaine or other psychomotor stimulants in a US-retrieval paradigm in the clinic.

\section{Potential Clinical Utility of Garcinol}

Garcinol is an exciting candidate for impairing drugmemory reconsolidation, as it can be safely administered in humans and has previously been demonstrated to inhibit reconsolidation of aversive (Maddox et al, 2013a) and appetitive (Monsey et al, 2016b) memories in rats after CS reactivation. Future studies may employ dose-response curves for the effects of garcinol to determine its range of efficacy. Importantly, the effects of garcinol on reconsolidation are long lasting, context-independent, reactivationdependent, and occur regardless of memory strength (Maddox et al, 2013a; Monsey et al, 2016b). One possible mechanism of action for garcinol's effect on reconsolidation is through an epigenetic action, as garcinol-induced inhibition of fear and cocaine reconsolidation has been demonstrated to correlate with an inhibition of retrieval-related acetylation of amygdalar histone H3 (Maddox et al, 2013a; Monsey et al, 2016a). In addition, the effect of garcinol on cocaine memory reconsolidation after CS retrieval can be reversed by concurrent administration of a histone deacetylase inhibitor (Monsey et al, 2016a). Garcinol has also been shown to inhibit both P300/CBP-associated factor (PCAF) and p300 HATs, with an increased affinity for the former (Balasubramanyam et al, 2004), and selective inhibition of p300/CBP HATs has impaired fear memory reconsolidation (Maddox et al, 2013b). The effect of garcinol on cocaine reconsolidation in the US-reactivation paradigm may be due to similar epigenetic mechanisms.

Garcinol should be investigated in clinical populations (Fuchs and McLaughlin, 2016) in order to assess its efficacy and therapeutic potential for relapse to substance use. Most clinical studies of reconsolidation and substance use in the literature have investigated the $\beta$-adrenergic antagonist propranolol with mixed results (Jobes et al, 2015; Lonergan et al, 2016; Pachas et al, 2015; Saladin et al, 2013; Zhao et al, 2011). However, preclinical investigations of garcinol and propranolol in a CS-reactivation paradigm have demonstrated that garcinol may be more effective at reducing reinstatement than propranolol (Dunbar and Taylor, 2016). Similar results were found with propranolol in a USreactivation paradigm in our lab (unpublished preliminary data). In addition, propranolol administration may actually be contraindicated in the presence of cocaine (Malbrain et al, 1994; Olson et al, 1983), which would be problematic when attempting the US-reactivation paradigm in humans. Specifically, several studies have reported that propranolol pretreatment exacerbates sensitivity to cocaine-induced toxicity and death (Catravas and Waters, 1981; Guinn et al, 1980; Smith et al, 1991; Tella et al, 1992; Williams et al, 2003) and potentiates the strength and duration of other cocaineinduced effects (Branch and Knuepfer, 1994; Fraker et al, 1995; Harris et al, 1996; Henning, 1993; Kiritsy-Roy et al, 1990; Lange et al, 1990; Ramoska and Sacchetti, 1985). The effect of garcinol on reconsolidation in humans should therefore be explored. 


\section{Future Avenues for Research on the US-Reactivation Paradigm}

Although the benefit of a single treatment with cocaine in the clinic during a US-reactivation procedure to treat addiction may far outweigh the costs of relapse, treatment providers and patients may be wary to support treatment that includes administration of the original drug of abuse. Therefore, it may also be of interest to investigate whether an alternative psychomotor stimulant or dopamine agonist could be used in lieu of cocaine during US reactivation. Other studies using different reconsolidation-like paradigms suggest this may be possible. For example, one study discussed above showed that administration of either methylphenidate or cocaine before several days of retrieval extinction inhibits the reconsolidation of multiple cocaine-paired cues (Luo et al, 2015), indicating that the dopamine agonist methylphenidate may be a sufficient substitute for reactivating a cocaine US memory. Subsequent research could examine whether methylphenidate followed by garcinol injection in a single treatment session would also impair the reconsolidation of multiple cocaine-paired cues in our paradigm.

Future avenues of research could investigate whether the effects of post-US-reactivation garcinol on reconsolidation persevere in self-administration models that have an even higher translational relevance to addiction. For example, after extended access to cocaine, animals show enhanced drug motivation and insensitivity to punishment (Milton and Everitt, 2010), which may provide intriguing insight into garcinol's treatment potential. In addition, if reconsolidation manipulations are to be used for treatment of psychiatric disorders, it is imperative that only the aberrant memories, rather than general memory functions, are disrupted. Thus, demonstrating that the memories of CSs that are unrelated to the reactivated US are spared in this paradigm would bolster the paradigm's translational relevance.

\section{Summary}

Overall, the present results demonstrate that garcinol treatment following cocaine reactivation inhibits reinstatement to multiple drug-paired cues in a model of rat cocaine self-administration. These findings suggest that clinical studies of reconsolidation examining the efficacy of the drug US-reactivation paradigm and post-reactivation garcinol may be beneficial and that memory reconsolidation-based treatments should be further explored as a potential therapy for psychopathologies including addiction.

\section{FUNDING AND DISCLOSURE}

This work was supported by DA015222 (to JRT), the Charles B.G. Murphy Fund, the Connecticut Department of Mental Health and Addiction Services, and the NSF Graduate Research Fellowship Program. The authors declare no conflict of interest.

\section{ACKNOWLEDGMENTS}

We thank Hayde Sanchez for assistance and Drs Stephanie M Groman, Melissa S Monsey, Mary M Torregrossa, and Stacey L Quick for helpful discussions.

\section{REFERENCES}

Balasubramanyam K, Altaf $M$, Varier RA, Swaminathan V, Ravindran A, Sadhale PP et al (2004). Polyisoprenylated benzophenone, garcinol, a natural histone acetyltransferase inhibitor, represses chromatin transcription and alters global gene expression. J Biol Chem 279: 33716-33726.

Bouton ME, Westbrook RF, Corcoran KA, Maren S (2006). Contextual and temporal modulation of extinction: behavioral and biological mechanisms. Biol Psychiatry 60: 352-360.

Branch CA, Knuepfer MM (1994). Causes of differential cardiovascular sensitivity to cocaine. I: Studies in conscious rats. J Pharmacol Exp Ther 269: 674-683.

Catravas JD, Waters IW (1981). Acute cocaine intoxication in the conscious dog: studies on the mechanism of lethality. J Pharmacol Exp Ther 217: 350-356.

Debiec J, Díaz-Mataix L, Bush DEA, Doyère V, LeDoux JE (2010). The amygdala encodes specific sensory features of an aversive reinforcer. Nat Neurosci 13: 536-537.

Díaz-Mataix L, Debiec J, LeDoux JE, Doyère V (2011). Sensoryspecific associations stored in the lateral amygdala allow for selective alteration of fear memories. J Neurosci 31: 9538-9543.

Doyère V, Debiec J, Monfils M-H, Schafe GE, LeDoux JE (2007). Synapse-specific reconsolidation of distinct fear memories in the lateral amygdala. Nat Neurosci 10: 414-416.

Dunbar AB, Taylor JR (2016). Inhibition of protein synthesis but not $\beta$-adrenergic receptors blocks reconsolidation of a cocaineassociated cue memory. Learn Mem 23: 391-398.

Fraker TD, Temesy-Armos PN, Brewster PS, Wilkerson RD (1995). Interaction of propranolol, verapamil, and nifedipine on the myocardial depressant effect of cocaine. J Cardiovasc Pharmacol 25: 579-586.

Fuchs RA, McLaughlin RJ (2016). Garcinol: a magic bullet of amnesia for maladaptive memories? Neuropsychopharmacology 42: 581-583.

Guinn MM, Bedford JA, Wilson MC (1980). Antagonism of intravenous cocaine lethality in nonhuman primates. Clin Toxicol 16: 499-508.

Harris GC, Hedaya MA, Pan WJ, Kalivas P (1996). beta-adrenergic antagonism alters the behavioral and neurochemical responses to cocaine. Neuropsychopharmacology 14: 195-204.

Henning RJ (1993). Cocaine significantly impairs myocardial relaxation. Crit Care Med 21: 575-585.

Jobes ML, Aharonovich E, Epstein DH, Phillips KA, Reamer D, Anderson $\mathrm{M}$ et al (2015). Effects of prereactivation propranolol on cocaine craving elicited by imagery script/cue sets in opioiddependent polydrug users: a randomized study. J Addict Med 9: 491-498.

Kiritsy-Roy JA, Halter JB, Gordon SM, Smith MJ, Terry LC (1990). Role of the central nervous system in hemodynamic and sympathoadrenal responses to cocaine in rats. J Pharmacol Exp Ther 255: 154-160.

Lange RA, Cigarroa RG, Flores ED, McBride W, Kim AS, Wells PJ et al (1990). Potentiation of cocaine-induced coronary vasoconstriction by beta-adrenergic blockade. Ann Intern Med 112: 897-903.

Lonergan MH, Saumier D, Tremblay J, Kieffer B, Brown TG, Brunet A (2016). Reactivating addiction-related memories under propranolol to reduce craving: a pilot randomized controlled trial. J Behav Ther Exp Psychiatry 50: 245-249.

Luo Y, Xue Y, Liu J, Shi H, Jian M, Han Y et al (2015). A novel UCS memory retrieval-extinction procedure to inhibit relapse to drug seeking. Nat Commun 6: 7675.

Maddox SA, Watts CS, Doyère V, Schafe GE (2013a). A naturallyoccurring histone acetyltransferase inhibitor derived from Garcinia indica impairs newly acquired and reactivated fear memories. PLoS ONE 8: e54463.

Maddox SA, Watts CS, Schafe GE (2013b). p300/CBP histone acetyltransferase activity is required for newly acquired and reactivated fear memories in the lateral amygdala. Learn Mem 20: 109-119. 
Malbrain ML, Neels H, Vissers K, Demedts P, Verbraeken H, Daelemans $\mathrm{R}$ et al (1994). A massive, near-fatal cocaine intoxication in a body-stuffer. Case report and review of the literature. Acta Clin Belg 49: 12-18.

Milton AL, Everitt BJ (2010). The psychological and neurochemical mechanisms of drug memory reconsolidation: implications for the treatment of addiction. Eur J Neurosci 31: 2308-2319.

Monsey MS, Gerhard DM, Duman RS, Taylor JR. Molecular and region specific effects of garcinol on cocaine-associated memory reconsolidation. Program No. 350.08/FFF5. Neurosci 2016 Abstr. San Diego, CA: Soc Neurosci, 2016a. Online.

Monsey MS, Sanchez H, Taylor JR (2016b). The naturally-occurring compound Garcinia indica selectively impairs the reconsolidation of a cocaine-associated memory. Neuropsychopharmacology 42: 587-597.

Nayak PK, Misra AL, Mulé SJ (1976). Physiological disposition and biotransformation of $(3 \mathrm{H})$ cocaine in acutely and chronically treated rats. J Pharmacol Exp Ther 196: 556-569.

Olson K, Benowitz NL, Pentel P (1983). Management of cocaine poisoning. Ann Emerg Med 12: 655-657.

Pachas GN, Gilman J, Orr SP, Hoeppner B, Carlini SV, Grasser EB et al (2015). Single dose propranolol does not affect physiologic or emotional reactivity to smoking cues. Psychopharmacology (Berl) 232: $1619-1628$.

Ramoska E, Sacchetti AD (1985). Propranolol-induced hypertension in treatment of cocaine intoxication. Ann Emerg Med 14: $1112-1113$.

Saladin ME, Gray KM, McRae-Clark AL, Larowe SD, Yeatts SD, Baker NL et al (2013). A double blind, placebo-controlled study of the effects of post-retrieval propranolol on reconsolidation of memory for craving and cue reactivity in cocaine dependent humans. Psychopharmacology (Berl) 226: 721-737.

Smith M, Garner D, Niemann JT (1991). Pharmacologic interventions after an LD50 cocaine insult in a chronically instrumented rat model: are beta-blockers contraindicated? Ann Emerg Med 20: $768-771$.

Sorg BA (2012). Reconsolidation of drug memories. Neurosci Biobehav Rev 36: 1400-1417.

Taylor JR, Olausson P, Quinn JJ, Torregrossa MM (2009). Targeting extinction and reconsolidation mechanisms to combat the impact of drug cues on addiction. Neuropharmacology 56 (Suppl 1): 186-195.

Tella SR, Korupolu GR, Schindler CW, Goldberg SR (1992). Pathophysiological and pharmacological mechanisms of acute cocaine toxicity in conscious rats. J Pharmacol Exp Ther 262: 936-946.

Torregrossa MM, Taylor JR (2013). Learning to forget: manipulating extinction and reconsolidation processes to treat addiction. Psychopharmacology (Berl) 226: 659-672.

Tronson NC, Taylor JR (2007). Molecular mechanisms of memory reconsolidation. Nat Rev Neurosci 8: 262-275.

Williams JB, Keenan SM, Gan Q, Knuepfer MM (2003). Hemodynamic response profile predicts susceptibility to cocaine-induced toxicity. Eur J Pharmacol 464: 189-196.

Zhao LY, Sun LL, Shi J, Li P, Zhang Y, Lu L (2011). Effects of $\beta$-adrenergic receptor blockade on drug-related memory reconsolidation in abstinent heroin addicts. Drug Alcohol Depend 118: 224-229.

Supplementary Information accompanies the paper on the Neuropsychopharmacology website (http://www.nature.com/npp) 